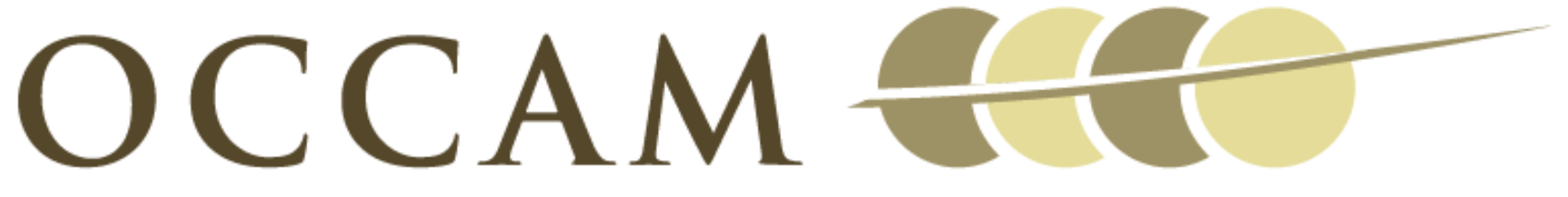

OXFORD CENTRE FOR COLLABORATIVE APPLIED MATHEMATICS

Report Number 09/26

Functional differential equations arising in cell-growth by

G.C. Wake and R.E. Begg

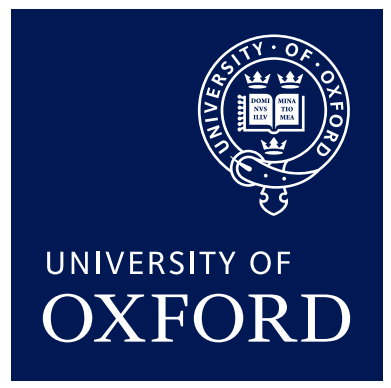

Oxford Centre for Collaborative Applied Mathematics Mathematical Institute 24 - 29 St Giles'

Oxford

OX1 3LB

England 



\title{
Functional differential equations arising in cell-growth
}

\author{
G. C. Wake*and R. E. Begg ${ }^{\dagger}$
}

July 31, 2009

\begin{abstract}
Non-local differential equations are notoriously difficult to solve. Cell-growth models for population growth of a cohort structured by size, simultaneously growing and dividing, give rise to a class of non-local eigenvalue problems, whose "principal" eigenvalue is the time-constant for growth/decay. These and other novel non-local problems are described and solved in special cases in this paper.
\end{abstract}

\section{Introduction}

Nonlocal calculus is relatively undeveloped. Thus occurs when cause and effect are separated in space, size, age, or time and give rise to functional differential equations. These problems frequently require unusual boundary and/or initial conditions to ensure they are well-posed - which would be expected for problems which come from modelling situations. Some well-known are

EXAMPLE. $y^{\prime}(t)=y(t-T)$ for all $t \in R, T>0$, which unlike the corresponding local equation when $T=0$ has a countably infinite number of linearly independent solutions

$$
B=\left\{e^{\lambda_{i} t}: i=1, \ldots n, \ldots\right\}
$$

where the $\lambda_{i}$ 's satisfy the transcendental equation

$$
\lambda=e^{-\lambda T}
$$

and so there is only one real solution in $B$.

This delay equation, which is well-known of course, needs to have an initial condition $y(t)=y_{0}(t),-T<t \leq 0$, to make it well-posed. Even more bizarre behaviour emerges if $T<0$, when the "future dictates the past".

EXAMPLE. $y^{\prime}(t)=y(\alpha t)$, for all $t \in R^{+}, \alpha>0$ also has an interesting solution set which is markedly different if $\alpha \gtrless 1$. This is a special case of the "pantograph equation". See [1] and [2]. If $y(0)=y_{0}$ is given, it is well-posed if $\alpha<1$. For $\alpha>1$, it has quite different behaviour.

\footnotetext{
${ }^{*}$ Corresponding author: Institute of Information and Mathematical Sciences, Massey University at Albany, Private Bag 102904, North Shore Mail Centre, Auckland, New Zealand. e-mail: g.c.wake@massey.ac.nz, Phone: +64(0) 94140800 Fax: +64 (0) 94418136

†University of Waterloo, Canada. e-mail rbegg@maths.uwaterloo.ca.
} 


\section{The cell-growth model}

It was well-known to biologists prior to the 1980's that cell-populations, structured by size ( $x$ which is synonymous here to DNA content) which evolve by simultaneously growing, dividing and dying, grow asymptotically towards a steady-size distribution (SSD) which has constant shape with increasing or decreasing size depending on the parameters. In a series of papers, for example [3] this was obtained mathematically by using a generalised Fokker-Planck equation.

$$
n_{t}=(D n)_{x x}-(g n)_{x}-\mu n+\alpha^{2} B(\alpha x) n(\alpha x, t)-B(x) n(x, t),
$$

for the evolving, with time $t$, of number density of cells $n(x, t)$, where $D$ is the dispersion coefficient, $g$ is the per-capita growth rate, $\mu$ is the per-capita death rate and $B(x)$ is the frequency of division of cells of size $\alpha x$ into $\alpha$ cells of size $x$, with $\alpha>1$. Although it does not require $\alpha$ to be an integer from a mathematical point of view, usually $\alpha=2$.

The equation (1) is usually accompanied by the zero-flux condition

$$
[D(x) n(x, t)]_{x=0}-g(0) n(0, t)=0, \quad t>0,
$$

and decay at infinity

$$
n(x, t), n_{x}(x, t) \rightarrow 0, \quad \text { as } \quad x \rightarrow \infty .
$$

The proof that there are SSD-like solutions is established beyond all doubt by observing that there are solutions of the form

$$
n(x, t)=e^{-\lambda t} y(x), \quad x, t>0
$$

where $\lambda, y(x)$ satisfy

$$
(D y)^{\prime \prime}(x)-(g y)^{\prime}(x)-\mu y+\alpha^{2} B(\alpha x)-B(x) y(x)+\lambda y=0, x>0,^{\prime}=\frac{d}{d x}
$$

with

$$
\left.\begin{array}{l}
\begin{array}{l}
y(x) \geq 0,(D y)^{\prime}(0)-(g y)(0)=0 \\
y(\infty)=y^{\prime}(\infty)=0
\end{array}
\end{array}\right\}
$$

where $y(\infty)$ denotes

$$
\lim _{x \rightarrow \infty} y(x)
$$

The first equation in (5) is that of a zero-flux condition.

Without loss of generality we can take $y(x)$ to be a probability density function, and so 


$$
\int_{0}^{\infty} y(x) \mathrm{d} x=1
$$

The problem in equations (4), (5), (6) are what we shall call a non-local, singular Sturm-Liouville eigenvalue problem. The question of the existence of SSD-like solutions becomes that of : "Is there an eigenvalue $\lambda$, and eigenfunction $y$ of this problem?" Further, is it unique within a multiplicative constant (equation (6) eliminates the multiplicity anyway).

\section{Results}

\subsection{Special cases}

$B(x)=b$ (a constant), with splitting at any size $x$. There is only one eigenvalue. Note that there will be other eigenfunctions but not with a mono-signed eigenfunction, which must satisfy

$$
\int_{0}^{\infty} y(x) \mathrm{d} x=0 .
$$

The eigenvalue is $\lambda=\mu-b(\alpha-1)$ with solution for $y(x)$ a Dirichlet series of the general form

$$
y(x)=\sum_{n=0}^{\infty} b_{n} e^{-k x^{n}},
$$

where $k, b_{n}$ are constants.

This function is just one peak and does not exhibit the two peaks formed in practice with data sets. This means that

$$
\mu \gtrless b(\alpha-1) \quad \text { ensures a } \begin{gathered}
\text { dying } \\
\text { growing }
\end{gathered} \text { cohort of cells. }
$$

It is interesting that this is independent of the growth rate $\mathrm{g}$ and dispersion rate $\mathrm{D}$. Which outcome you want depends if the cells are ones you want (muscle cells etc.) or ones you do not (tumour cells).

\subsection{General}

$B(x)$ Without the requirement of having a mono-signed eigenfunction it is possible to prove there is a countable number of eigenvalues by restricting the domain to $x \in$ $(0, L)$ with $L$ arbitrarily large. To get two bumps in the one mono-signed eigenvalue we require something like $B(x)=b \delta(x-\ell)$, where $\delta(x-\ell)$ is the Dirac-delta function and $\ell$ is the cell-splitting size.

We expect this result to apply as $L \rightarrow \infty$ and conjecture this is so. See [4]. 


\subsection{More general non-local eigenvalue problems}

The above can be extended to problems like

$$
\left.\begin{array}{l}
-y^{\prime \prime}(x)+a y^{\prime}(x)+b y(x)=\lambda y(g(x)), 0<x<\infty \\
y(0)=0, \quad y(\infty)=0,
\end{array}\right\}
$$

and $g(x)$ is a continuous function mapping $[0, \infty)$ to itself. Letting $z=1-$ $e^{-x}, Y(z)=y(x)$, we can then transform the problem (9) into a problem on a finite domain $[0,1]$, use the Green's function for the compact domain to obtain a compact operator and so the problem can be re-stated as a functional integral equation using the Green's function of the operator on the left-hand side of equation (9). This again shows that there is a countable number of eigenvalues, and each eigenvalue has finite multiplicity.

\subsection{Stability}

In most of the above cases we are able to prove that the monosigned SSD behaviour is globally attracting. That is, $n(x, t) \sim C e^{-\lambda t} y(x)$ for large time irrespective of the sign of $\lambda$, and the difference decays faster as $t$ increases.

\section{Discussion}

Recently we came across the non-local heat equation - not based on a physically real problem which appears well-posed, but is actually not finite for arbitrarily small time:

$$
\left.\begin{array}{l}
U_{x x}(1-x, t)=U_{t}(n, t), \quad 0<x<1, \quad t>0 \\
U(0, t)=U(1, t)=0, \\
U(x, 0) \text { given. }
\end{array}\right\}
$$

Then $U(x, t)$ is unbounded as $t$ increases. This shows like all the above problems, that there are astounding unexpected results coming from this and that the earlier nonlocal problems which appear deceptively simple.

ACKNOWLEDGEMENT. The publication was completed while an author (GCW) was a Visiting Fellow at OCCAM (Oxford Centre for Collaborative Applied Mathematics) under support provided by Award No. KUK-C1-013-04, made by King Abdullah University of Science and Technology (KAUST).

\section{References}

[1] J. R. Ockendon, and A. B. Taylor, The dynamics of a current collection system for a locomotive. Proceedings of the Royal Society London A(322), pp. 447-468, (1971).

[2] L. Fox, D. F. Mayers, J. R. Ockendon, and A. B. Taylor, On a functional differential equation, J.Inst.Math.Appl. 8, pp. 271-307, (1971). 
[3] G. C. Wake, S. Cooper, H-Y. Kim, and B. van Brunt, Functional differential equations for cell-growth models with dispersion, Comm.Appl.Anal., 4, pp. 561-573, (2000).

[4] B. Basse, G. C. Wake, D. J. N. Wall, and B. van Brunt, On a cell-growth model for plankton, Math.Med. \& Bio: A Journal of the IMA, 21, pp. 49-61, (2004). 


\section{RECENT REPORTS}

2009

02/09 Diffusion and permeation in binary solutions Peppin

03/09 On the modelling of biological patterns with mechanochemical Moreo models: insights from analysis and computation Gaffney

Garcia-Aznar

Doblare

04/09 Stability analysis of reaction-diffusion systems with timemdependent coefficients on growing domains

Madzvamuse

Gaffney

Maini

05/09 Onsager reciprocity in premelting solids

Peppin

Spannuth

Wettlaufer

06/09 Inherent noise can facilitate coherence in collective swarm motion

Yates et al.

07/09 Solving the Coupled System Improves Computational Efficiency of the Bidomain Equations

Southern

Plank

Vigmond

Whiteley

08/09 Model reduction using a posteriori analysis

Whiteley

09/09 Equilibrium Order Parameters of Liquid Crystals in the LaudauDe Gennes Theory

10/09 Landau-De Gennes theory of nematic liquid crystals: the OseenFrank limit and beyond

Majumdar

Majumdar

Zarnescu

11/09 A Comparison of Numerical Methods used for Finite Element Modelling of Soft Tissue Deformation

Pathmanathan

Gavaghan

Whiteley

12/09 From Individual to Collective Behaviour of Unicellular Organisms: Recent Results and Open Problems

Xue

Othmer

Erban

13/09 Stochastic modelling of reaction-diffusion processes: algorithms for bimolecular reactions

Erban

Chapman

14/09 Chaste: a test-driven approach to software development for phys-

Pitt-Francis et al. iological modelling

15/09 Block triangular preconditioners for PDE constrained optimization

Rees

Stoll 
16/09 From microscopic to macroscopic descriptions of cell migration on growing domains

Baker

Yates

Erban

17/09 The Influence of Gene Expression Time Delays on Gierer-

Seirin Lee Meinhardt Pattern Formation Systems

Gaffney

Monk

18/09 Analysis of a stochastic chemical system close to a sniper bifurca-

Erban et al. tion of its mean field model

19/09 On the existence and the applications of modified equations for Zygalakis stochastic differential equations

20/09 Pebble bed: reflector treatment and pressure velocity coupling

Charpin et al.

21/09 A finite difference method for free boundary problems

Fornberg

22/09 Tangent unit-vector fields: nonabelian homotopy invariants and

Majumdar the Dirichlet energy

Robbins

Zyskin

23/09 Morphological instability of a nonequilibrium icecolloid interface

Peppin

Majumdar

Wettlaufer

24/09 The effect of polar lipids on tear film dynamics

Aydemir

Breward

Witelski

25/09 Preconditioning for active set and projected gradient methods as

Stoll semi-smooth Newton methods for PDE-constrained optimization with control constraints

Wathen

Copies of these, and any other OCCAM reports can be obtained from:

Oxford Centre for Collaborative Applied Mathematics Mathematical Institute

24 - 29 St Giles'

Oxford

OX1 3LB

England

www.maths.ox.ac.uk/occam 